Ensayo

\title{
Curriculum: de la edad media al siglo XIX
}

\section{Curriculum: from the Middle Ages to the 19th century}

\author{
Domingo Pérez Brito \\ Maestría en Currículum \\ Universidad de San Carlos de Guatemala \\ https://orcid.org/0000-0003-3783-1951 \\ perezbrito42@yahoo.com
}

\section{Referencia}

Pérez Brito, D. (2021). Curriculum: de la edad media al siglo XIX. Revista Guatemalteca de Educación Superior, 4(2), 61-76. DOI: https://doi.org/10.46954/revistages.v4i2.63

Recibido: 15/02/2021

Aceptado: 04/04/2021

\section{Resumen}

Un elemento central del curriculum es el llamado paradigma o enfoque como factor clave que orienta la estructuración y el diseño de carreras profesionales. En este sentido, el paradigma es la categoría o noción que permea y da forma a una determinada propuesta educativa. Como tales, los paradigmas surgen y se desarrollan en contrapartida a las diferentes coyunturas históricas y que en el campo educativo dan respuesta a las necesidades y demandas económicas y sociales del momento. Es así como de la edad media al siglo XIX, surgen y, eventualmente, se complementan los paradigmas: teológico, humanista, científico y nacional que acompañan las transformaciones económicas y políticas que determinan y definen a los sistemas educativos y, por lo tanto, a las propuestas curriculares. Cada uno de los paradigmas curriculares se analiza de acuerdo al entorno económico en el que surge tomando en cuenta los acontecimientos históricos y la visión del mundo y del hombre que hegemoniza cada momento o coyuntura. Con ello 


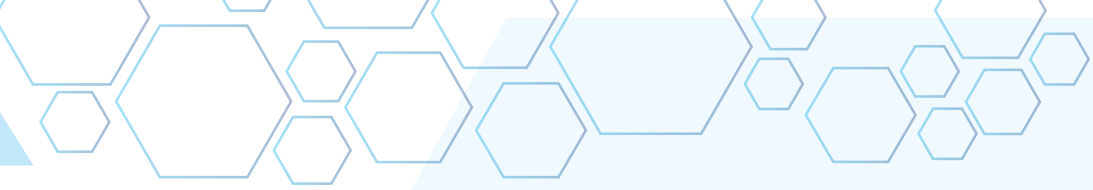

Palabras clave:

curriculum, paradigma, evolución, enfoque, disciplina se intenta reflejar la influencia del modelo económico respecto a la definición, carácter y naturaleza del sistema educativo y, por lo tanto, del curriculum y de cómo la escuela y el proyecto curricular responden a las necesidades y demandas del entorno económico.

\section{Abstract}

A central element of the curriculum is the so-called paradigm or approach as a key factor that guides the structuring and design of professional careers. In this sense, the paradigm is the category or notion that permeates and shapes a specific educational proposal. As such, paradigms emerge and develop in counterpart to the different historical conjunctures and that in the educational field respond to the economic and social needs and demands of the moment. Thus, from the Middle Ages to the 19th century, the paradigms emerge and eventually complement each other: theological, humanistic, scientific and national that accompany the economic and political transformations that determine and define educational systems and, therefore, the curricular proposals. Each of the curricular paradigms is analyzed according to the economic environment in which it arises, taking into account historical events and the vision of the world and of the man that hegemonizes each moment or conjuncture. This is intended to reflect the influence of the economic model on the definition, character and nature of the educational system and, therefore, the curriculum and how the school and the curricular project respond to the needs and demands of the economic environment.

\section{Introducción}

El currículo o curriculum es un término que se origina del latín y puede significar carrera, ruta o camino y se utiliza también como sinónimo de plan de estudios. En general, dicho término implica un movimiento permanente y progresivo que se refiere al recorrido académico de un estudiante y, por extensión, al recorrido profesional y vital de una persona. Curriculum se origina del verbo curro que significa carrera, además de currere que significa caminar: ambos términos se refieren a la carrera 
estudiantil y a la carrera de la vida. Los seres humanos caminamos y corremos dentro de las avenidas que nos provee el sistema educativo (Casarini, 2007).

Asimismo, un apartado fundamental en el diseño curricular es el paradigma curricular que se define o se instituye en el marco de la tradición teórica y de los objetivos educativos de determinado momento histórico. Es así como a partir de la edad media hasta el siglo XIX, se identifican los enfoques o paradigmas: teológico, humanista, científico y nacional. En currículo y en el presente caso, el término paradigma se utiliza en el sentido señalado por Kuhn:

El paradigma representa las realizaciones científicas universalmente reconocidas que, durante cierto tiempo, proporcionan modelos de problemas y soluciones a una comunidad científica. Cada revolución científica modifica la perspectiva histórica de la comunidad que la experimenta y ese cambio de perspectiva influye en las investigaciones, las publicaciones y los libros de texto (Kuhn, 1971).

En vista de lo ya indicado, el propósito del presente trabajo es la revisión lde las principales características de los paradigmas curriculares que surgen, evolucionan e influyen los sistemas educativos que, gradualmente, evolucionan de satisfacer las demandas de la elite económica a la incorporación creciente de la clase media y la clase trabajadora.

\section{Desarrollo}

\section{La edad media}

La edad media es una larga etapa histórica cuyos inicios están marcados por el final del imperio romano, el surgimiento de las llamadas ciudades-estado y lo que posteriormente sería la iglesia católica. Es una etapa jalonada por los sucesivos asedios de mongoles, vikingos, el desencadenamiento de las cruzadas, las peregrinaciones y el inicio de las exploraciones hacia oriente. 
En este periodo, el viaje de Marco Polo que llega hasta China donde se queda un largo tiempo, se convierte en un caso especial, especialmente porque al regresar a Italia, tiene tiempo para contar sus fantásticas vivencias (Polo, 2006).

Otro hechos y acontecimientos relevantes como el surgimiento del sacro imperio de Carlomagno en el centro de Europa, la creación de las primeras universidades que no obstante estar en manos de las órdenes religiosas, son centros de transmisión y desarrollo del conocimiento. En la parte final, el siglo XV, testifica la toma de Constantinopla por parte de los musulmanes, la multiplicación de los viajes y exploraciones fruto y resultado concreto del desarrollo marítimo y que conlleva el descubrimiento de América.

La edad media se caracteriza por su definida, firme e inamovible estructura en clases sociales claramente diferenciadas: en la parte alta, la nobleza que incluye a los altos dirigentes de la iglesia y a los grandes terratenientes. Dueños absolutos del medio de riqueza predominante: la tierra. En la parte baja, los siervos sobre los que recae la responsabilidad de hacer producir la tierra con trabajo semigratuito para beneficio de la nobleza. En medio, especialmente en las ciudades, se agrupan comerciantes y artesanos. Una pequeña clase media que incluye a médicos, abogados, profesores y otros miembros de las profesiones liberales.

Respecto a las ideas y las creencias, en la edad media se concreta y se arraiga la visión teológica del mundo y de la vida tomando como base la tradición judeo-cristiana en Europa: prevalece el dualismo entre lo terrenal y lo celestial. Se considera lo divino (Dios) como el centro y motor del universo. Como efecto derivado y especifico es la idea de que los planetas giran alrededor de la tierra: lo que posteriormente se conoció como paradigma geocéntrico. Entre los pensadores que moldean la mentalidad medieval está San Agustín, Santo Tomás de Aquino y las ideas adaptadas de los griegos: Aristóteles, Ptolomeo, Galeno, entre otros. 
Como se ha indicado, la creación de riqueza tiene como fuente la posesión de la tierra por lo que el eje principal de la producción se encuentra en el campo. La nobleza y la iglesia son los principales beneficiarios cuya riqueza se asienta en el trabajo servil de los campesinos atados legalmente al campo. En este escenario, Asimov indica que en el siglo $X$, se produce un enorme adelanto tecnológico con la introducción del estribo y del arado, que influye de manera decisiva en un espectacular aumento de la producción. El aumento productivo genera excedentes que a su vez permiten el surgimiento y el mantenimiento de ejércitos permanentes, además de un pequeño sector que se dedicará exclusivamente a la práctica del arte y al estudio de la ciencia (Asimov, 2011).

En el campo educativo y curricular prevalece el paradigma teológico: la realidad y el conocimiento se explican en base a las creencias religiosas. El Estado y el sistema educativo están bajo control ideológico de la iglesia y su carácter aristocrático se define por funcionar al servicio de la nobleza. El sistema de educación superior se estructura en base al trívium y el quadrivium. El primero centrado en el lenguaje: gramática, retórica y lógica. El segundo centrado en los números: aritmética, geometría, astronomía y música. En este esquema, el primer título para el estudiante es la licenciatura en las siete "artes liberales" (trívium y quadrivium) al que se agrega la ética, la metafísica y la filosofía natural. Estos estudios son preparatorios para otros de mayor nivel que ya son las carreras profesionales: teología, derecho y medicina (Burke, 2002).

En contrapartida, la capacitación en los oficios conocidos como artes mecánicas, se encuentran en manos de los gremios, estructurados igualmente en estricto orden jerárquico. En general, el curriculum o plan de estudios ya se define como "el conjunto multianual de estudios" que debe transitar un estudiante. La cualidad de control, orden y eficiencia de la que hace gala se hereda de la visión educativa de jesuitas y después los calvinistas, expertos administradores educativos: las diferencias religiosas no les impiden la construcción de sistemas y modelos educativos sostenidos por valores asentados en la jerarquía social y el autoritarismo. 
Tabla No.1 El curriculum de enfoque teológico

\begin{tabular}{|c|c|c|c|c|}
\hline Ciclos & Tiempo & Enfoque & Asignaturas & Grado \\
\hline I & 3 años & Lenguaje & $\begin{array}{l}\text { - Gramática } \\
\text { - Retórica } \\
\text { - Lógica }\end{array}$ & - Bachiller \\
\hline \multirow[t]{2}{*}{ II } & 4 años & Matemática & $\begin{array}{l}\text { - Aritmética } \\
\text { - Geometría } \\
\text { - Astronomía } \\
\text { - Música }\end{array}$ & \\
\hline & & Complemento & $\begin{array}{l}\text { - Ética, } \\
\text { - Metafísica } \\
\text { - Filosofía natural }\end{array}$ & - Licenciatura \\
\hline Tercer nivel & & Profesional & $\begin{array}{l}\text { - Teología } \\
\text { - Derecho canónico } \\
\text { - Derecho civil } \\
\text { - Medicina }\end{array}$ & $\begin{array}{l}\text { - Maestría } \\
\text { - Doctorado }\end{array}$ \\
\hline
\end{tabular}

Fuente: Burke, 2002.

\section{El renacimiento}

La edad media llega a su fin en medio de grandes acontecimientos como descubrimientos y viajes de exploración hacia lugares considerados como inaccesibles. La conquista del mar por parte de marinos españoles y portugueses: Cristóbal Colón descubre América, Núñez de Balboa lo hace con el mar pacífico, Vasco da Gama llega finalmente a la India, Fernando de Magallanes y Juan Sebastián Elcano, completan la vuelta al mundo. Además, la aparición y el uso de la pólvora, la brújula y la imprenta trastocan el entorno económico, político y social. Adicionalmente, el statu quo es estremecido por la aparición, lenta y tímida, de la teoría heliocéntrica que cuestiona y refuta la estructura de creencias y valores prevalecientes. La suma de exploraciones, descubrimientos y avances tecnológicos terminan definitivamente con una etapa de la humanidad marcada por el atraso y la ignorancia. El cambio de mentalidad impacta a las élites y a la población en general.

En el renacimiento, al contrario de la edad media, el hombre se convierte en el eje y centro del universo. Además, gana importancia la práctica y la experiencia como fuentes de conocimiento, la naturaleza y la realidad física y material se 
convierten en modelos y factores de aprendizaje. Como lo señala Bramly, el método del renacimiento es "adquirir, en forma racional y científica, el dominio de los medios". (Bramly, 2015) La innata curiosidad del ser humano gana impulso y se promueve el afán y el ansia de aprender, divulgar y enseñar al mismo tiempo que se generaliza el estudio y la comprensión del entorno social y natural.

Junto a las universidades se posicionan las academias como centros alternativos de difusión y creación del conocimiento. El pequeño sector intelectual $y$, en especial, los artistas tienen la posibilidad de enriquecerse con su trabajo y, gradualmente, adquieren autonomía frente a los mecenas tradicionales: los grandes señores de la nobleza. Como personajes renacentistas se puede incluir a Leonardo, Loyola, Erasmo y Vives, entre otros.

El modelo económico se mantiene, en términos generales, aunque la creación de riqueza asentado en el campo se complementa con la actividad y el crecimiento de las ciudades y el consiguiente fortalecimiento de comerciantes, financistas y de los artesanos organizados en gremios sanos. Esta tendencia que se manifiesta en las ciudades marca el paulatino crecimiento de un influyente sector que evolucionaria hacia el surgimiento de la burguesía como clase social hegemónica. Es pues, un momento de gran renovación que además del arte y la ciencia, transforma la vida económica y social de la población.

Además de los grandes inventos y descubrimientos, el renacimiento es sacudido también por otros eventos de gran trascendencia como sería el caso de la Reforma y la ContraReforma. En 1517, Lutero se hace visible con sus 95 tesis; en 1536, Calvino publica su Institución de la Religión Cristiana; en 1542, Roma crea la Congregación de la Suprema Inquisición. Ambos procesos arrastran a Europa a una etapa de conflictos religiosos que colocan a la orden del día las purgas, masacres, expulsiones y exilios que afectan a la población y a la élite: técnicos, científicos, artistas; también sufren las consecuencias. 
La Reforma impulsa la interpretación personal de la biblia y el vínculo con Dios sin intermediarios. Este hecho deriva directamente hacia una mayor importancia de los idiomas nacionales y, consecuentemente, en la necesidad de alfabetizar a la población: para que los feligreses puedan leer la biblia es imprescindible que aprendan a leer en su propio idioma. El latín, como idioma hegemónico, llegaría a su fin. En el aspecto educativo, los cambios socioeconómicos, obligan una vez más al sistema educativo a adaptarse a las demandas del entorno. En concreto, los jesuitas diseñan la ratio studiorum o plan de estudios con una clara orientación humanista: el primer nivel se enfoca en las humanidades y la gramática. Un segundo nivel comprende cursos de sagrada escritura, lengua hebrea, teología escolástica, casos de conciencia, filosofía, filosofía moral y matemáticas (Farrel, 1970).

La influencia humanista de la Ratio studiorum, no obstante mantener la estructura básica de los estudios medievales, se manifiesta en los fines y sus valores implícitos, la visión de la formación integral con el planteamiento de un modelo educativo que se propone abarcar las ciencias exactas, las ciencias humanas, las bellas artes y la cultura física: es la manifiesta integración de conocimientos científicos y humanísticos, el ideal del hombre la educación holista. La visión del ser humano como una totalidad, no una suma de sus partes.

Tabla No. 2 El curriculum de enfoque humanista

\begin{tabular}{|c|c|c|c|}
\hline Ciclos & Tiempo & Énfasis & Asignaturas \\
\hline Estudios inferiores & 5 años & $\begin{array}{l}\text { Gramática } \\
\text { humanismo clásico }\end{array}$ & $\begin{array}{l}\text { y- Gramática elemental, } \\
\text { - Gramática intermedia } \\
\text { - Gramática superior. } \\
\text { - Humanidades } \\
\text { - Retorica }\end{array}$ \\
\hline II & 7 años & Filosofía y teología & $\begin{array}{l}\text { - Sagradas escrituras } \\
\text { - Hebreo } \\
\text { - Teología escolástica } \\
\text { - Casos de conciencia } \\
\text { - Filosofía } \\
\text { - Filosofía moral } \\
\text { - Matemáticas }\end{array}$ \\
\hline
\end{tabular}

Fuente: Farrel, 1970. 


\section{La revolución científica}

El renacimiento y la reforma protestante se constituyen en el prolegómeno de un escenario más dinámico que genera cambios científicos, políticos y económicos. La revolución científica (la ilustración) origina el gran desarrollo de la ciencia, la tecnología y el predominio de las ciencias básicas. La revolución científica es pronto acompañada por las revoluciones burguesas con la francesa a la cabeza. Mientras en el campo económico se inicia lo que posteriormente se conocería como revolución industrial que acelera la producción de bienes cuyo eje se basa en la industria y el comercio frente al agro que se mantiene como productor de materia prima. El alcance y la profundidad de estas revoluciones trastoca la base material y espiritual de la sociedad. Es una etapa de gran esplendor y de transformaciones que arraigan la noción de que el progreso es permanente.

Reflejo de las ideas prevalecientes es el surgimiento de la visión racional y pragmática de la vida y del esfuerzo individual como garantía del logro de metas y objetivos igualmente personales. Frente a la visión religiosa de la realidad se erige la visión laica y la propuesta, siempre cuestionada, de la separación entre Estado e iglesia. Los miembros educados de la élite social se convierten en activos difusores de las "nuevas ideas" económicas, políticas y sociales con lo cual preparan el camino para una época de mayores trasformaciones en los siglos XVIII y XIX.

El desarrollo científico transforma la producción de riqueza que se mueve del campo a la ciudad, de la tierra al capital y, de nuevo, la producción genera excedentes. El crecimiento de la población, de las ciudades y el aumento del intercambio económico provoca la destrucción del sistema de corporaciones que funcionan como monopolios. Es patente la necesidad de alinear el sistema político con los nuevos aires económicos: el sistema feudal se convierte en freno para el desarrollo económico y el gobierno en manos de la aristocracia tiene los días contados. Las revoluciones burguesas crean las condiciones para la consolidación de los Estados nacionales, la creación de escuelas y universidades en manos del Estado, la consolidación 
de la burguesía como clase social, el crecimiento de la población asalariada, el debilitamiento de la nobleza y de la iglesia, entre otros factores.

La escuela y el curriculum se adaptan a los cambios con el surgimiento del paradigma científico que enfatiza las ciencias exactas y naturales que obtienen reconocimiento social y dan lugar a la jerarquización de las disciplinas: las ciencias exactas aumentan su prestigio frente a las ciencias sociales. La renovación tecnológica se basa en el método científico con lo cual la investigación y la experimentación se convierten en los ejes centrales para la adquisición y creación de conocimiento.

Junto a la escuela y la universidad se multiplica la creación de jardines botánicos, aulas de experimentación, laboratorios, bibliotecas, observatorios, museos, etc. Los científicos y académicos se agrupan en comunidades que les permiten discutir sus trabajos, los temas de moda, los avances y las nuevas tendencias. Se privilegia la aplicación del conocimiento en los centros educativos que permiten a los estudiantes actuar, tocar, ejecutar, diseñar, interactuar, etc. Es la consagración del conocimiento práctico y útil.

La influencia de las ideas se extiende de los países centrales hacia los países periféricos. En Guatemala, por ejemplo, la revolución científica y la ilustración influye en los pequeños círculos de profesores universitarios abiertos a los cambios y a la renovación del conocimiento, que ya no están cómodos y a gusto con el modelo escolástico que aún prevalece. Es el caso de Liendo y Goicochea que propugna por la reforma universitaria y se aboca al diseño de un nuevo plan de estudios cuyos postulados de transformación educativa no tendrían resultados tan inmediatos (Tate, 1978). 
Tabla No. 3 El curriculum de enfoque científico

\begin{tabular}{|c|l|c|l|}
\hline No. & \multicolumn{1}{|c|}{ Cátedras existentes } & No. & \multicolumn{1}{|c|}{ Cátedras nuevas } \\
\hline 1 & Cánones & 1 & Retórica \\
\hline 2 & Leyes & 2 & Lógica y metaphísica \\
\hline 3 & Theología prima & 3 & Mathematica necesaria para Física \\
\hline 4 & Theología moral & 4 & Phísica experimental \\
\hline 5 & Medicina & 5 & Anathomía \\
\hline 6 & Instituta & 6 & Medicina \\
\hline 7 & Theologia de Escoto & 7 & Theologia moral \\
\hline 8 & Philosofia de Escoto & 8 & Derecho canónico \\
\hline 9 & Philosofia Thomista & 9 & Instituciones, leyes \\
\hline 10 & Philosofia & 10 & Escritura sagrada \\
\hline 11 & Lengua (suspensa) & 11 & Theologia dogmática \\
\hline & & 12 & Lenguas de indias \\
\hline
\end{tabular}

Fuente: Tate, 1978.

\section{El Estado-nación}

Con la revolución francesa se conforman los estados nacionales y los grandes imperios europeos. En el primer caso, la necesidad económica agrupa a sociedades con un pasado común en territorios claramente definidos y diferenciados de los vecinos que, eventualmente, son considerados como enemigos y como competidores. Como lo señala Bartra:

La nación es uno de los modos en que se estructura la sociedad, como producto de un proceso de coagulación de un pasado histórico y que se expresa en la comunidad de lengua, territorio, vida económica y cultura (Bartra, 1973).

En el segundo caso, se constituyen los grandes imperios europeos: Gran Bretaña, Francia, Austria-Hungría, Rusia, Turquía. Esta realidad histórica define la distribución de influencias y el establecimiento de colonias en Asia, África y América Latina. El 
modelo colonial somete pueblos con el uso de la fuerza militar y se mantiene por la necesidad del control comercial en beneficio de las nacientes industrias. Estos imperios prevalecen hasta el desencadenamiento de la primera guerra mundial que provoca su desaparición o debilitamiento. Con los Estados-nación se fortalece la ideología del patriotismo y del nacionalismo que serían una de las causas de las guerras mundiales del siglo XX.

La visión y el pensamiento pragmático se reafirma con el surgimiento de la visión racional y la razón como grandes orientadores de la actividad humana, florece el individualismo, el interés personal prevalece ante el interés colectivo y el egoísmo humano adquiere aceptación social: primero y ante todo el beneficio personal y sí se logra el éxito económico siempre queda la práctica de la caridad. En un contexto de desarrollo científico y de evolución cultural florecen las teorías de Newton, Marx y Freud, entre otros, que impactan en las creencias, ideas y visiones de las elites y de los ciudadanos y cuyo influjo todavía es palpable en las esferas económicas, políticas y científicas.

El ambiente de dinamismo científico y tecnológico gradualmente se transforma en la primera revolución industrial que acelera y multiplica la producción de bienes y servicios, especialmente con las industrias del carbón y del acero. La producción de energía descansa en la máquina de vapor previo al surgimiento de la energía eléctrica que se convierte asimismo en una industria. Se desarrolla la economía de mercado y el predominio de la ley de la oferta y la demanda. La burguesía se convierte en la clase dirigente, la nobleza pasa a un segundo plano, se recupera la alianza entre Estado e iglesia y la democracia se considera el ideal de gobierno. El capital se constituye en el factor básico del sistema económico y los avances tecnológicos generan la sensación de progreso material interminable. A pesar del desarrollo material, se mantienen incólumes las desigualdades económicas y sociales, situación que alimenta las guerras y los ciclos de violencia.

En el sector de la educación, la formación universitaria pasa a una nueva etapa con la multiplicación de universidades y el surgimiento de las grandes tradiciones: la francesa con énfasis 
en las disciplinas científicas y humanísticas, financiamiento y control estatal; la alemana enfocada hacia la investigación y la experimentación; la inglesa que introduce el financiamiento privado y empresarial. Asimismo, se produce la consolidación de las disciplinas científicas con una clara diferenciación entre ciencias exactas y ciencias sociales, la formación privilegiada de la élite económica que accede a los estudios universitarios mientras que la clase media y trabajadora se orienta a los estudios técnicos y comerciales.

Los Estados nacionales se abocan a la construcción de países homogéneos y centralizados que delegan en el sistema educativo el reto de construir y consolidar la "cultura nacional", por lo tanto, los planes de estudio evolucionan hacia contenidos específicos: historia, geografía, idioma, literatura, religión, etc., con énfasis en lo nacional. (Lundgren, 1997) Se consolida el paradigma curricular nacional y patriótico. El objetivo central es la formación del ciudadano especifico de cada país, en teoría un país homogéneo étnica y culturalmente; lo que requiere un modelo educativo nacional y la implantación de mitos, símbolos y héroes que la población haga suyos.

Tabla No. 4 El curriculum de enfoque nacional

\begin{tabular}{|c|l|}
\hline No. & \multicolumn{1}{|c|}{ Materias } \\
\hline 1 & Filosofía \\
\hline 2 & Matemática \\
\hline 3 & Ciencias naturales \\
\hline 4 & Historia (nacional) \\
\hline 5 & Geografía (nacional) \\
\hline 6 & Idioma (nacional) \\
\hline 7 & Religión (oficial) \\
\hline 8 & Valores y moral (cultura nacional) \\
\hline
\end{tabular}

Fuente: Lundgren, 1997. 


\section{Conclusión}

El desarrollo y la constante ampliación de los sistemas educativos y del curriculum obedece a la necesidad de "transmitir y reproducir" los valores y anhelos de una determinada época histórica. Por lo tanto, el curriculum es un instrumento que estructura la selección, organización y evaluación de objetivos, contenidos, prácticas y relaciones del proceso de enseñanzaaprendizaje. La cualidad de control del curriculum se hereda de la edad media y se afianza en las subsiguientes etapas históricas con lo cual se consolidan las nociones básicas de orden y eficiencia como ejes del curriculum.

En el desarrollo histórico del curriculum se pueden identificar tendencias como las siguientes: El contexto socioeconómico define y orienta los objetivos y contenidos de los planes de estudio que evolucionan consecutivamente en los paradigmas: teológico, humanista, científico y nacional. La gradual evolución de un enfoque culto, clásico y humanístico hacia un enfoque científico, experimental y de aplicación del conocimiento. Las incesantes ampliaciones del "árbol del conocimiento" conforman nuevas disciplinas como la química, literatura, historia, farmacia, economía, estadística, agricultura e ingeniería. El conocimiento se expande y es necesaria su clasificación a través de bibliotecas, museos, archivos, enciclopedias, atlas, diccionarios, etc. 


\section{Referencias}

Asimov, Isaac. (2011) La Alta Edad Media. Las edades oscuras. Madrid: Alianza. 328 pp.

Bartra, Roger. (1973) Breve diccionario de sociología marxista. México: Grijalbo.

Burke, Peter. (2002) Historia social del conocimiento. Barcelona: Paidós. 321 pp.

Bramly, Serge. (2015) Leonardo da Vinci. Buenos Aíres: El Ateneo. 447 pp.

Casarini, Martha. (2007) Teoría y diseño curricular. México: Trillas. 230 pp.

Farrel, Allan P. (1970) The Jesuit ratio studiorum of 1599. Washington: Conference of major superiors of jesuits. 149 pp. Recuperado de: https://3eh4ot43gk9g3h1uu7edbbf1wpengine.netdna-ssl.com/wp-content/uploads/ documents/2019/09/ratio-studiorum-1599.pdf

Lundgren, Ulf P. (1997) Teoría del curriculum y escolarización. Madrid: Morata. 127 pp.

Polo, Marco. (2006) Los viajes de Marco Polo narrados por él mismo. Buenos Aires: Claridad. 207 pp.

Kuhn, Thomas S. (1971) La estructura de las revoluciones científicas. México: FCE. 360 pp.

Tate Lanning, John. (1978) La Ilustración en la Universidad de San Carlos. Guatemala: Editorial Universitaria. 579 pp. 


\section{Sobre el autor}

\section{Domingo Pérez Brito}

Es Licenciado en Letras por la Universidad de San Carlos de Guatemala, Master en Educación Bilingüe Intercultural por la Universidad Rafael Landívar y Master en Currículo por la Universidad del Valle de Guatemala. Ha laborado en el sector público, privado y no gubernamental. Ha escrito sobre literatura, educación y currículo. Actualmente trabaja como profesional de desarrollo curricular en el Departamento de Asesoría y Orientación Curricular.

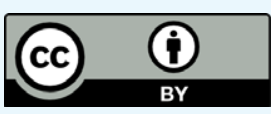

Este texto está protegido por una licencia CreativeCommons 4.0.

Usted es libre para compartir, copiar y redistribuir el material en cualquier medio o formato y adaptar el documento, remezclar, transformar y crear a partir del material para cualquier propósito, incluso comercialmente, siempre que cumpla la condición de atribución: usted debe reconocer el crédito de una obra de manera adecuada, proporcionar un enlace a la licencia, e indicar si se han realizado cambios. Puede hacerlo en cualquier forma razonable, pero no de forma tal que sugiera que tiene el apoyo del licenciante o lo recibe por el uso que hace. 